\title{
Search for muon neutrinos from GRBs with the ANTARES neutrino telescope
}

\author{
Silvia Celli* \\ On behalf of the ANTARES Collaboration \\ Gran Sasso Science Institute, Viale Francesco Crispi 7, 67100 L'Aquila, Italy \\ INFN-Sezione di Roma, P.le Aldo Moro 2, 00185 Roma, Italy \\ E-mail: silvia.celli@gsi.infn.it
}

\begin{abstract}
ANTARES is the largest operational neutrino telescope in the Northern hemisphere, located in the deep water of the Mediterranean Sea, offshore Toulon. One of its main scientific goals concerns the identification of hadronic astrophysical accelerators through the detection of high-energy neutrinos. Among these sources, Gamma-Ray Bursts (GRBs) constitutes promising candidates because they are the most bright sources in the Universe. Their transient nature allows to drastically reduce the expected background when both a temporal and spatial correlation with the observed gamma-ray prompt emission is required. Cosmic neutrinos could be produced in the interaction between accelerated protons and intense radiation fields in the jet. Two different approaches have been adopted in the search: a stacked analysis with a sample of GRBs observable using the full ANTARES data set (from 2008 to 2016) and an individual search from some of the brightest GRBs (with gamma-ray fluence greater than $10^{-4} \mathrm{erg} / \mathrm{cm}^{2}$ ) occurred in the same time period. The methods and the results of these searches for muon neutrinos are here presented. The stacking analysis allows to constrain the contribution to the diffuse flux of neutrinos from this population of sources. In the bright GRB analysis, instead, the internal shock and the photospheric scenarios have been investigated and limits in the parameter space of the fireball model are derived individually. Since no events have been detected in spatial and temporal coincidence with GRBs in any of the searches, upper limits on neutrino fluence are derived both for individual bright sources and for the GRB population sample.
\end{abstract}

35th International Cosmic Ray Conference - ICRC2017

10-20 July, 2017

Bexco, Busan, Korea

${ }^{*}$ Speaker. 


\section{Introduction}

The first detection of cosmic neutrinos [1] with still unidentified astrophysical sources is one of the main open questions of modern astrophysics. Despite the lack of a clear multi-messenger connection, different interpretations of the signal have come from the theoretical side, addressing both the galactic and the extra-galactic scenario. Many efforts have been carried out from the experimental side as well in order to constraint the contribution to this signal of different source classes. Among them, Gamma-Ray Bursts (GRBs) represents valuable candidates, being the most powerful catastrophic events of the Universe. In baryonic jet models, neutrinos are expected to be produced in the interaction among accelerated protons and the jet radiation field. Thanks to their properties of electrical neutrality, stability and weak interaction, neutrinos are able to reach the Earth undeflected and unabsorbed, directly pointing to the emission site. Both muon and electron neutrinos emerge from $p \gamma$ interaction: however, the most adequate event sample for astronomical purposes is represented by the muon sample, given the very good angular resolution that can be achieved, particularly in water. Therefore, a search from muon neutrinos from GRBs is here presented, using the ANTARES data set from 2008 to 2016: sources below the detector horizon are here considered in order to strongly suppress the atmospheric muon background, while an unbinned likelihood analysis is performed to distinguish the signal from the irreducible atmospheric neutrino background. A model-dependent search, based on both the internal shock and of the photospheric scenarios of the GRB fireball model is performed. Previous searches in both the ANTARES [2] and the IceCube [3] telescopes did not succeed in the identification of neutrino events spatially and temporally correlated with the detected gamma-ray emissions. The paper is structured as follows: an introduction to the theoretical models connecting gamma-rays and neutrinos in GRBs is introduced in Sec. 2. The analysis method for an optimized search is then discussed in Sec. 3, in order to present in Sec. 4 an individual search from four bright GRBs and in Sec. 5 a diffuse search performed though the stacking technique. In these sections, constraints on the physics of GRBs are derived as well. Conclusions are then given in Sec. 6.

\section{Gamma-ray bursts modeling}

The most accepted scenario describing the emission of the detected gamma-rays (in the so called prompt phase of the GRB) is the fireball model [5]. A central engine injects in the interstellar medium plasma shells with different velocities: particle acceleration is occurring when a faster shell catches up with a slower one. Both protons and electrons are thought to be accelerated in this process, while gamma-rays are mainly produced through inverse Compton scattering over the accelerated electrons. Protons interacting with the radiation field can produce both gamma-rays and neutrinos through the $\Delta^{+}$resonant channel:

$$
p+\gamma \rightarrow \Delta^{+} \rightarrow\left\{\begin{array} { l } 
{ p + \pi ^ { 0 } } \\
{ n + \pi ^ { + } }
\end{array} \rightarrow \left\{\begin{array}{l}
\pi^{0} \longrightarrow \gamma+\gamma \\
n \longrightarrow p+e^{-}+\bar{v}_{e} \\
\pi^{+} \longrightarrow \mu^{+}+v_{\mu} \\
\mu^{+} \longrightarrow e^{+}+v_{e}+\bar{v}_{\mu}
\end{array}\right.\right.
$$


In the internal shock (IS) scenario the radius of shell collisions is located at [6]

$$
\mathrm{R}_{I S}=2 \frac{c t_{v a r}}{1+z} \Gamma^{2} \sim 10^{13}\left(\frac{t_{v a r}}{0.01 \mathrm{~s}}\right)\left(\frac{\Gamma}{10^{2.5}}\right)^{2}\left(\frac{1}{1+z}\right) \mathrm{cm}
$$

where $t_{\text {var }}$ represents the variability time of the GRB emission, $\Gamma$ is the jet Lorentz factor and $z$ is the source redshift. In the photospheric ( $\mathrm{PH})$ scenario, instead, the collision radius is located at a lower distance from the central engine where the jet is still optically thick to gamma-rays, respectively at

$$
\mathrm{R}_{P H}=\frac{\mathrm{L}_{i s o} \sigma_{T}}{8 \pi m_{p} c^{3}} \Gamma^{-3} \sim 10^{11}\left(\frac{\mathrm{L}_{\text {iso }}}{10^{52} \mathrm{erg} / \mathrm{s}}\right)\left(\frac{\Gamma}{10^{2.5}}\right)^{-3} \mathrm{~cm}
$$

where $L_{i s o}$ is the isotropic luminosity of the burst, $\sigma_{T}$ is the Thomson cross section and $m_{p}$ is the proton mass. The radius of emission strongly affects both the energy range and the normalization of the neutrino expected fluence: the IS scenario predicts neutrinos mainly above $100 \mathrm{TeV}$, while the PH scenario introduces a low-energy component below $10 \mathrm{TeV}$.

Neutrino spectra are computed individually for each GRB: in the IS case, the numerical code NeuCosmA [7] provides spectra for individual neutrino flavors, while in the PH case the analytical description from [8] was adopted.

\section{Analysis methods}

The event selection proceeds through the application of an angular cut, a temporal cut and a cut on the quality of the track reconstruction. The first two cuts are applied in order to get a coincidence with the detected gamma-ray emission: a cone of aperture $\alpha=10^{\circ}$ is defined around the burst position, as identified by a given satellite. Although the ANTARES angular resolution for muon events is about $0.4^{\circ}$ at $E_{v}=10 \mathrm{TeV}$, the size of the angular window is enlarged in order to account for the satellite uncertainty on the burst position, which might reach value of the order of several degrees (especially when Fermi GBM is providing the burst coordinates); however, given the transient nature of the sources, such a large window is not prohibitive in terms of background. The temporal window is selected as the burst T90, which is the time during which $90 \%$ of the total fluence is detected, plus a symmetric extension of width $30 \% \mathrm{~T} 90$ which accounts for both the satellite timing uncertainty and the unknown delay between the gamma-ray and the neutrino emission inside the prompt phase of the GRB, plus a symmetric extension of $\pm 2 \mathrm{~s}$ to account for the time of light propagation from the satellite to the ANTARES site and for ANTARES uncertainty in the data acquisition. Finally, the analysis is based on an unbinned method that relies on the evaluation of a test statistic $\mathrm{Q}$ in a pseudo-experiment procedure defined as:

$$
Q=\max _{\mu_{s}^{\prime} \in\left[0 ; n_{\text {tot }}\right]}\left(\sum_{i=1}^{n_{\text {tot }}} \log \frac{\mu_{s}^{\prime} S\left(\alpha_{i}\right)+\mu_{b} B\left(\alpha_{i}\right)}{\mu_{b} B\left(\alpha_{i}\right)}-\mu_{s}^{\prime}\right)
$$

where $n_{t o t}$ is the number of simulated events in each measurements at an angular distance $\alpha_{i}$ from the source position, both for the background only hypothesis and for the signal plus background one. The test statistic is an extended maximum likelihood ratio, defined from the signal probability density function $S(\alpha)$ and the background probability density function $B(\alpha)$. The signal angular 
distribution is obtained through Monte Carlo simulations, realized specifically for each GRB, accurately describing the ANTARES conditions at the time of the GRB trigger, while the background distribution is assumed flat in the solid angle considered in the search. The number of expected background event $\mu_{b}$ in the temporal and angular window of the search is evaluated from off-time data, while the expected number of signal events $\mu_{s}^{\prime}$ is obtained in the likelihood maximization. Pseudo-experiments are performed at different quality cuts: the final reconstruction quality cut is selected as the one that maximizes the individual Model Discovery Potential (MDP) of each GRB, defined as the probability of the signal hypothesis at a given significance level $\sigma$.

\section{Individual search: bright GRBs}

A search for neutrinos from bright GRBs is performed, as extensively treated in [9]: four sources have been selected with gamma-ray fluence greater than $10^{-4} \mathrm{erg} / \mathrm{cm}^{2}$ in the time period from 2008 to 2013. The selection yielded GRB080916C, GRB110918A, GRB130427A and GRB130505A. Both the IS and the PH scenarios are investigated for neutrino emissions: in this analysis, a standard value for the bulk Lorentz factor is assumed $\Gamma=316$ for each GRB as well as a baryon loading equal to $f_{p}=10$. In order to increase the sensitivity to the low-energies predicted by the PH model, a special data sample is used in this case: it includes unfiltered data stored during the GRB alert, received through GCN notices [10], as well as unfiltered data buffered before the alert message reception. The overall size of the unfiltered data stored amounts to about 2 minutes, in such a way that they cover the majority of the burst duration. The IS analysis is instead performed through the standard filtered data set. Given the optimization technique described in Sec. 3, the cuts yielding the maximum $3 \sigma$ MDP were applied to unblinded data: no neutrinos were detected in the defined temporal and spatial windows of the search. The absence of signal allows to derive 90\% C.L. upper limits to the neutrino fluence, shown individually for each GRB in Fig. 1(a) for the IS model and in Fig. 1(b) for the PH one, in the energy range where $90 \%$ of the signal is expected.

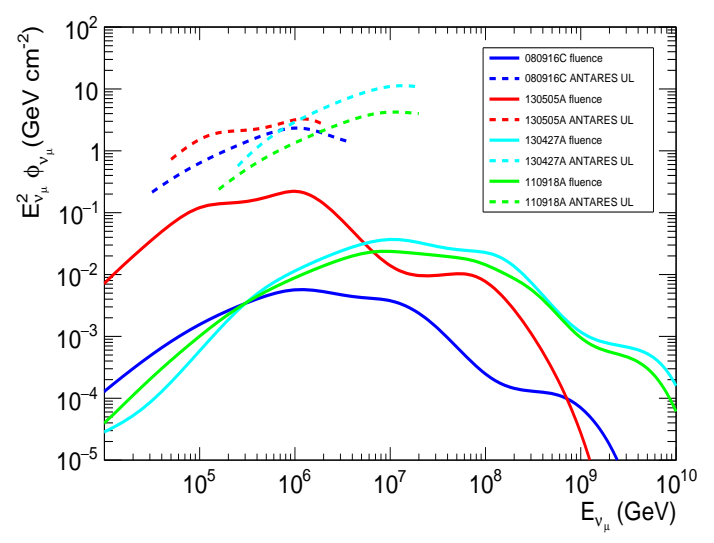

(a)

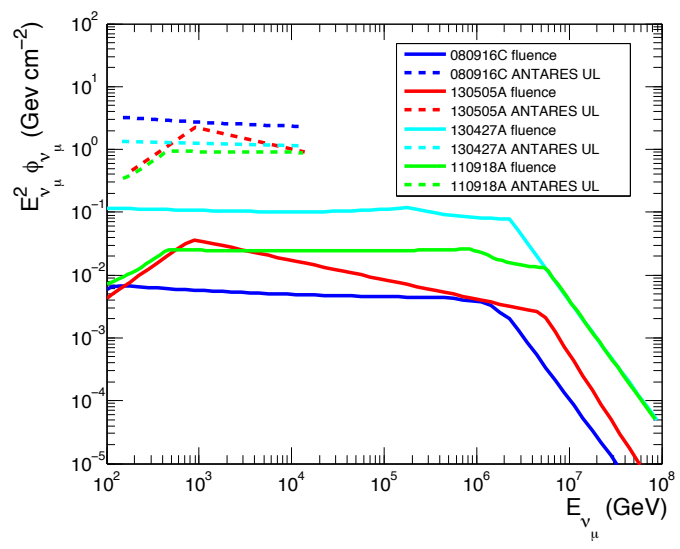

(b)

Figure 1: Expected $v_{\mu}+\bar{v}_{\mu}$ fluences (solid lines) and ANTARES 90\% C.L. upper limits (dashed lines) on the selected GRBs, in the energy band where $90 \%$ of the signal is expected to be detected, for the IS (a) and the PH (b) models. 


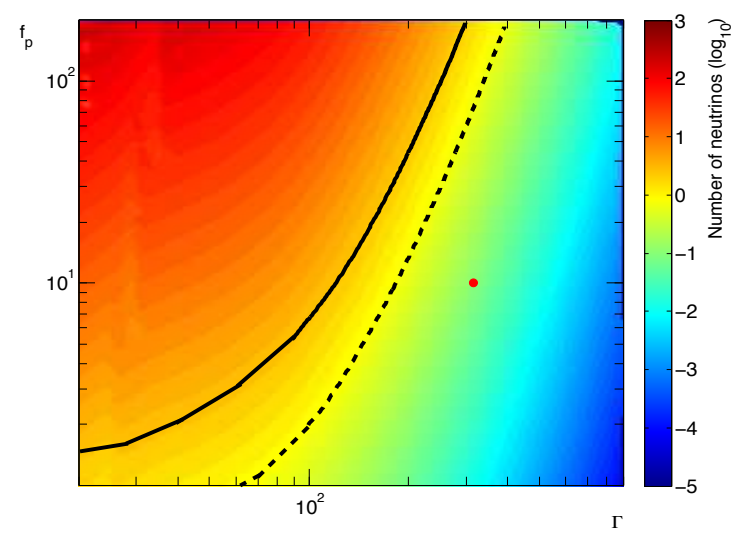

(a)

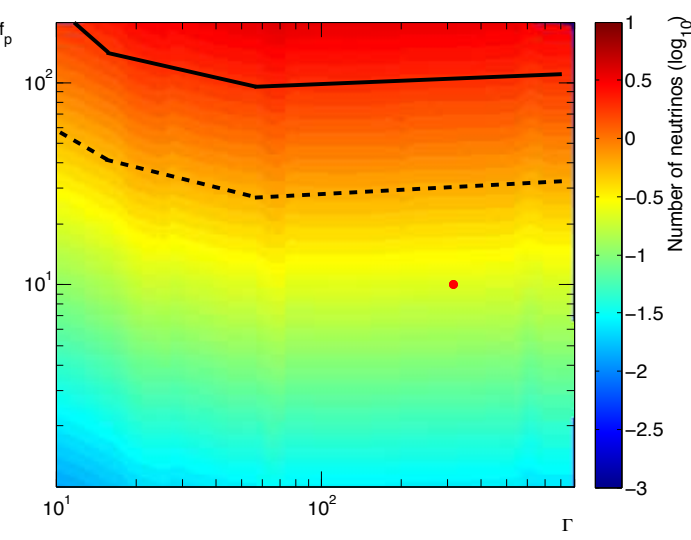

(b)

Figure 2: Constraints on the $\Gamma-f_{p}$ plane. The solid (dashed) black line corresponds to the exclusion limits at 90 (50)\% C.L. The red dot shows the benchmark values $f_{p}=10$ and $\Gamma=316$. (a) IS constraints on GRB130505A. (b) PH constraints on GRB130427A.

\subsection{GRB model constraints}

Given the null results discussed above in the search of neutrinos in angular and temporal coincidence with the gamma-ray emission detected from the bright GRBs, it is possible to derive constraints on the jet parameters affecting neutrino emissions, $\Gamma$ and $f_{p}$ for instance. The most stringent 90\% C.L. limit on the IS model is derived for GRB130505A and it is shown in Fig. 2(a), while the PH model is best constrained in the case of GRB130427A, as shown in Fig. 2(b). Low Lorentz factor and high baryonic loading are generally excluded, but still most of the parameter space is available. A different procedure will be adopted in the following stacking analysis: in order to leave the baryonic content as the main free parameter of the model, the bulk Lorentz factor of each burst is inferred through its correlation with the isotropic luminosity.

\section{Quasi-diffuse search: the stacking method}

The full sample of long GRBs (T90 $\geq 2$ s) observable with ANTARES in the years 20082016 was considered: a source selection is performed starting from the Swift and Fermi catalogs, completed through information available on the GCN. GRBs below the ANTARES horizon at the trigger time and with the detector in stable data-taking conditions are considered. Moreover, a stringent selection on the burst spectrum was applied, since a large extrapolation is needed to move from gamma-rays to the expected neutrinos: therefore, GRBs whose gamma-ray spectrum is fitted as a simple power law are excluded. This procedure resulted in 462 GRBs, whose position in the equatorial sky is shown in Fig. 3, where also the detected gamma-ray fluence is shown.

The internal shock scenario is here assumed as signal model. Moreover, a linear correlation among the burst isotropic luminosity and the bulk Lorentz factor is assumed, as derived in [11]: such a correlation reads as

$$
\Gamma=249\left(\frac{L_{i s o}}{10^{52} \mathrm{erg} / \mathrm{s}}\right)^{0.30}
$$




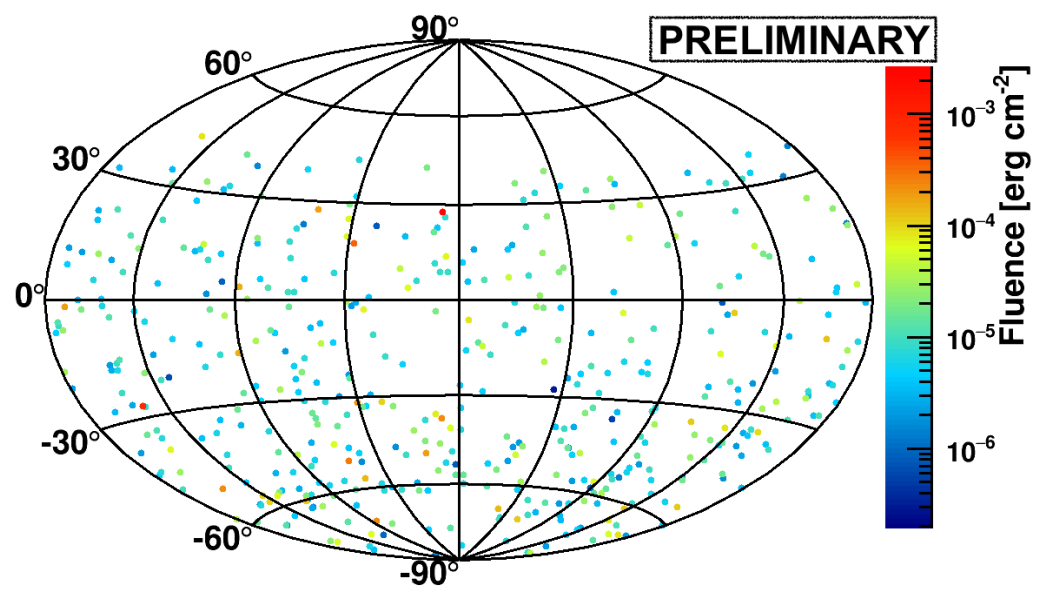

Figure 3: Sky map of the GRBs selected for the stacking analysis, in equatorial coordinates: the color code represents the measured gamma-ray fluence.

However, since the isotropic luminosity depends on the redshift and since most of the GRBs in the sample miss this information, we start assigning each burst a redshift by uniformly sampling a distribution of redshifts, as collected by the Swift satellite from long GRBs detected between 2005 and 2017, with the constraint that the resulting $L_{i s o}$ is among $10^{49}$ and $10^{53} \mathrm{erg} / \mathrm{s}$. Then $\Gamma$ is inferred through Eq. 5.1: an uncertainty of 50\% should be considered in the estimation of the Lorentz factor. The expected neutrino spectrum computed through $\operatorname{Neu} \operatorname{CosmA}$, assuming a baryon fraction $f_{P}=10$ and the $L_{i s o}-\Gamma$ correlation presented in Eq. 5.1, is shown in Fig.4(a), where the single contributions from each GRB are also shown. With this expected signal, the method described in Sec. 3 is then applied, complemented by the introduction of a statistical penalty factor that accounts for the increased size of the sample: indeed, in the stacking technique, the summed contribution from all sources is considered in the test statistic evaluation, yielding a diffuse flux of events. Given the per burst individual $M D P_{i}$, which is the maximum MDP obtainable among different track quality cuts for a single GRB at a given trial factor, the total MDP obtained when including in the search $N_{G R B s}$ is defined as

$$
\operatorname{MDP}\left(N_{G R B s}\right)=1-\prod_{i=1}^{N_{G R B S}}\left(1-M D P_{i}\right)
$$

The total MDP as a function of the number of GRBs included in the search is given in Fig. 4(b).

\subsection{GRB population constraints}

The analysis shows that at a $3 \sigma$ significance level the MDP reaches its maximum value of $11.5 \%$ for 254 stacked sources. However, when including all the GRBs of the sample, the MDP decreases only to $11.1 \%$ : given the moderate MDP reduction, it is preferable to consider the whole sample of 462 GRBs in the search for a neutrino signal. This results in a total livetime of the analysis equal to 11.2 hours. After the unblinding of data at the optimal cuts, no event is found in angular and temporal coincidence with the prompt gamma-ray emission of any of the GRBs. The $90 \%$ C.L. upper limit on the neutrino fluence $E^{2} F_{v}(E)$ stands a factor 18 above the expectations, as 


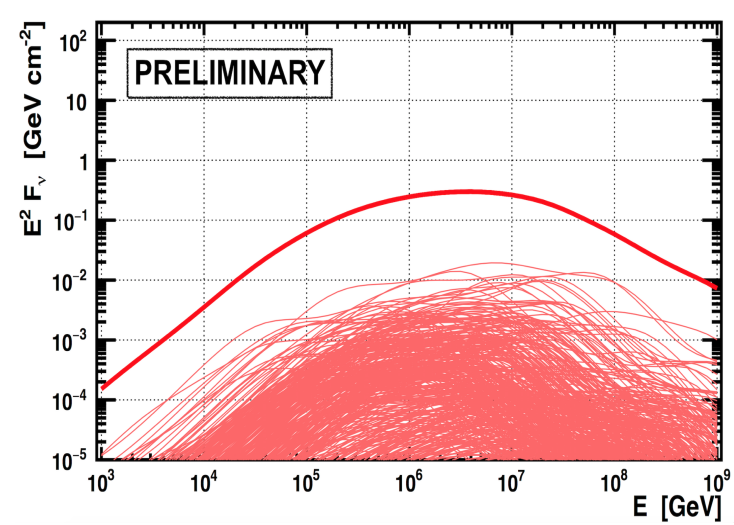

(a)

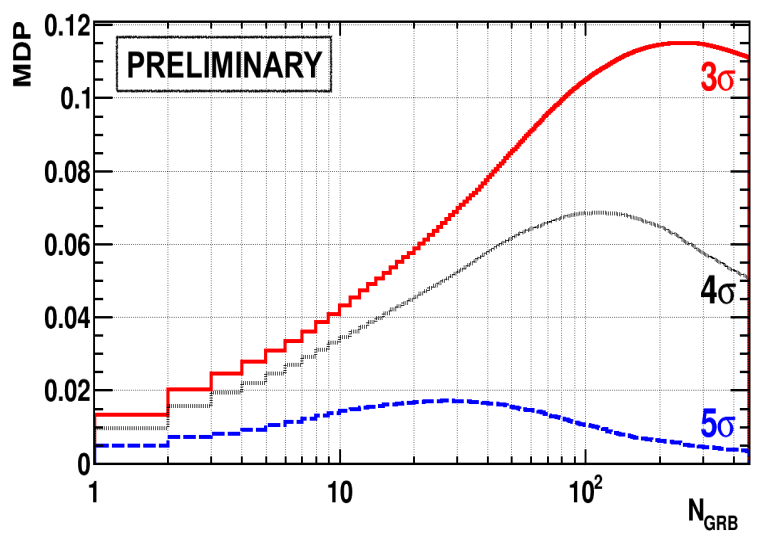

(b)

Figure 4: (a) Expected $v_{\mu}+\bar{v}_{\mu}$ fluences from the 462 GRBs of the stacking analysis (thin lines) and summed contribution (thick line), according to the IS modeling of the NeuCosmA code. (b) MDP of the stacking analysis as a function of the number of sources included.

shown in Fig. 5: the fluence interval where 90\% of the signal is expected ranges between 0.45 and $5.2 \mathrm{GeV} / \mathrm{cm}^{2}$ in the energy range from $5 \times 10^{4}$ to $8 \times 10^{6} \mathrm{GeV}$. The fluence limit can be translated into a limit on the quasi-diffuse flux from the GRB population: considering $N_{G R B s}$ over an average number of 667 long GRBs detected per year, the diffuse flux is

$$
E^{2} \phi_{v}(E)=\sum E^{2} F_{v}(E) \frac{1}{4 \pi} \frac{667 y r^{-1}}{N_{G R B s}}
$$

The $90 \%$ C.L. on the diffuse flux ranges between $1.6 \times 10^{-9}$ and $1.9 \times 10^{-8} \mathrm{GeV} \mathrm{cm}^{-2} \mathrm{~s}^{-1} \mathrm{sr}^{-1}$. Moreover, these results directly translate into constraints on the baryonic content of the jets, given that neutrino fluence linearly scales with $f_{p}$ : the limit equals to $f_{p} \leq 180$ at $90 \%$ C.L. and to $f_{p} \leq 89$ at $68 \%$ C.L. in GRB jets.

The Lorentz factor of the jet is one of the most critical microphysics parameters of the jet for the neutrino spectrum. Propagating the uncertainties in the relation $L_{i s o}-\Gamma$ affects significantly the shape of the neutrino spectrum: reducing the $\Gamma$ factor, increase the flux of neutrinos at low energies $(1-100 \mathrm{TeV})$ and vice versa. A $50 \%$ increase in the $L_{\text {iso }}-\Gamma$ relation worsen the limit by a factor 124 , while a $50 \%$ decrease starts to be constrained by the ANTARES limits. As a result, limits on the baryonic content are also strongly affected: in the optimistic scenario (decrease), the limit on $f_{p}$ reaches 10 at $90 \%$ C.L., which is a quite restrictive value for scenarios of CR proton acceleration in GRB jets. On the other hand, the limit soften to $f_{p} \leq 1240$ (90\% C.L.) in case the correlation is increased.

\section{Conclusions}

Through the technique defined in Sec. 3, nine years on ANTARES data were considered for a search of muon neutrinos in time and space coincidence with the prompt emission of 462 GRBs. Both an individual search from bright GRBs and a population study were performed, through an optimization technique based on the maximization of the Model Discovery Potential. However, 
no events passed the selection criteria, yielding $90 \%$ C.L. upper limits on the expected neutrino fluence and constraints on the parameters affecting the physical emission mechanism of GRBs. Limits and constraints are consistent with previous searches in ANTARES [2,9] and IceCube [3].

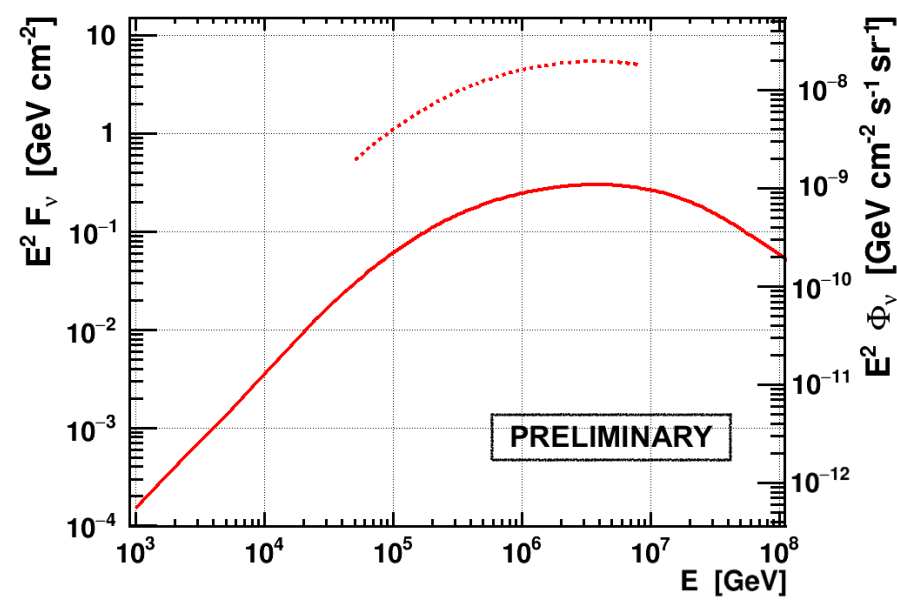

Figure 5: Expected $v_{\mu}+\bar{v}_{\mu}$ fluence (left axis) and diffuse flux (right axis) from the 462 GRBs of the stacking analysis (red solid line) and ANTARES $90 \%$ C.L. upper limits (dashed line), in the energy band where $90 \%$ of the signal is expected to be detected.

\section{References}

[1] M. G. Aartsen et al., Evidence for High-Energy Extraterrestrial Neutrinos at the IceCube Detector, Science 342 (2013) [arXiv:1311.5238]

[2] S. Adrian-Martinez et al., Search for muon neutrinos from gamma-ray bursts with the ANTARES neutrino telescope using 2008 to 2011 data, Astron. Astrophys. 559 (2013) [arXiv:1307.0304]

[3] M. G. Aartsen et al., Extending the search for muon neutrinos coincident with gamma-ray bursts in IceCube data [arXiv:1702.06868]

[4] M. Ageron at al., ANTARES: the first undersea neutrino telescope, Nucl. Instrum. Meth. A 656 (2011) 11-38 [arXiv:1104.1607]

[5] T. Piran, Gamma-ray bursts and the fireball model, Phys. Rept. 314 (1999) 575-667 [arXiv:9810256]

[6] M. Bustamante, P. Baerwald, K. Murase and W. Winter, Neutrino and cosmic-ray emission from multiple internal shocks in gamma-ray bursts, Nat. Com. 6 (2015) 6783 [arXiv:1409.2874]

[7] S. Hummer, P. Baerwald and W. Winter, Neutrino Emission from Gamma-Ray Burst Fireballs, Revised, Phys. Rev. Lett. 108 (2012) [arXiv:1112.1076]

[8] B. Zhang and P. Kumar, Model-dependent high-energy neutrino flux from Gamma-Ray Bursts, Phys. Rev. Lett. 110 (2013) 12 [arXiv:1210.0647]

[9] A. Albert et al., Search for high-energy neutrinos from bright GRBs with ANTARES, Mon. Not. Roy. Astron. Soc. 469 (2017) 906 [arXiv:1612.08589]

[10] https://gcn.gsfc.nasa.gov/gcn3_archive.html

[11] J. Lu, Y. C. Zou, W. H. Lei, B. Zhang, Q. Wu, D. X. Wang, E. W. Liang and H. J. Lu, Lorentz factor isotropic luminosity/energy correlations of gamma-ray bursts and their interpretation, The Astrophysical Journal 751 (2012) 49 [arXiv:1109.3757] 